\title{
HYPERMAXIMALITY OF CERTAIN OPERATORS ON LIE GROUPS ${ }^{1}$
}

\author{
I. E. SEGAL
}

We show that any polynomial in the infinitesimal generators of a Lie group is essentially hypermaximal symmetric (and so has a spectral resolution), when suitably interpreted as an operator on a Hilbert space, provided it is formally symmetric and commutes with all the infinitesimal generators. Such operators are frequently of use in investigations of the unitary representations of Lie groups. The requirement that the polynomial commute with the infinitesimal generators cannot be eliminated, unless some alternative restriction is put on the polynomial (for example, on its degree, as in $[2]^{2}$ ); this is shown by an unpublished example due to von Neumann.

Let $G$ be a connected Lie group with Lie algebra $\mathcal{L}$ (of right-invariant infinitesimal transformations) and let $\mathcal{E}$ be the enveloping algebra of $\mathcal{L}$ (see for example [1]). If $U$ is a (strongly) continuous unitary representation of $G$ on a Hilbert space $\mathcal{F}$, there exists (see [2]) an associated representation $d U$ of $\varepsilon$ by operators on $\mathfrak{F C}$ with domain $\mathcal{D}$ consisting of all sums of vectors of the form $\int U(a) x f(a) d a$, with $x \in \mathcal{H}, f$ a function of class $C^{\infty}$ on $G$ and vanishing outside a compact set (the integral being taken in the strong sense relative to the left-invariant Haar measure on $G$ with element $d a$ ), and characterized by the property that if $X \in \mathcal{L}$, then $i d U(X)$ is essentially hypermaximal symmetric ( $T$ is such an operator if $T^{*}=\left(T^{* *}\right)$, and $\exp \left(i t(d U(X))^{*}\right)=U(\exp (t X))$ for all real $t$. An element of $\mathcal{E}$ will be called "symmetric" if it is invariant under the unique real linear operaton on $\varepsilon$ which takes any monomial $\alpha X_{1} X_{2} \cdots X_{r}$ ( $\alpha$ complex, the $X_{i}$ in $\mathcal{L}$ ) into $(-1)^{r} \bar{\alpha} X_{r} \cdots X_{2} X_{1}$.

TheOREM. Let $p$ be a symmetric polynomial in the center of the enveloping algebra of the connected Lie group $G$, and let $U$ be a strongly continuous unitary representation of $G$ on the complex Hilbert space $\mathfrak{H C}$. Then $d U(p)$ is essentially hypermaximal symmetric on the domain $\mathcal{D}$ defined above.

Putting $d U(p)=T$, it suffices (see [4]) to show that $T+i I$ and $T$ $-i I$ have dense ranges. The proof which follows for the case of $T+i I$ applies also to $T-i I$. Let $\mathcal{H}$ be the orthogonal complement of the

Received by the editors June 1, 1951.

${ }^{1}$ This work was sponsored in part by the Office of Naval Research.

${ }^{2}$ Numbers in brackets refer to the references at the end of the paper. 
range of $T+i I$, so that $y \in \mathcal{X}$ means that $(T u, y)=-i(u, y)$, for $u \in \mathcal{D}$. Now $T$ commutes with the $U(a), a \in G$, for, following a method used in [3], if $X \in \mathcal{L}$ and $W(\alpha)=U(\exp (\alpha X)) T U(\exp (-\alpha X))-T$, then a computation given in [3] shows that when $u \in \mathcal{D}$ and $v \in \mathcal{H C}$, $d(W(\alpha) u, v) / d \alpha$ exists and vanishes, $-\infty<\alpha<\infty$, so that $(W(\alpha) u, v)$ $=0$ for all $\alpha$, whence $T$ commutes with the $U(\exp (\alpha X))$, and every $U(a)$ is a finite product of the $U(\exp (\alpha X))$. It follows easily that $\mathscr{X}$ is invariant under the $U(a)$, so that the projection $P$ with range $\mathscr{N}$ commutes with the $U(a)$.

Now if $u \in \mathcal{D}$ has the form $u=\int U(a) x f(a) d a$, then as continuous linear operations commute with strong integration, it results that $P u=\int P U(a) x f(a) d a=\int U(a) x^{\prime} f(a) d a$ with $x^{\prime}=P x$. Thus $D$ is invariant under $P$ and if $u$ is arbitrary in $\mathcal{D}$, then $P u=u^{\prime}$ say is in both $\mathscr{D}$ and $\mathcal{H}$, so that

$$
\left(T u^{\prime}, u^{\prime}\right)=-i\left(u^{\prime}, u^{\prime}\right) \text {. }
$$

Now $\left(u^{\prime}, u^{\prime}\right)$ is real, and so also is $\left(T u^{\prime}, u^{\prime}\right)$, for $i d U(X)$ is symmetric for $X \in \mathcal{L}$, which together with the invariance of $\mathscr{D}$ under the $d U(X)$ implies that $\alpha X_{1} X_{2} \cdots X_{r}+(-1)^{r} \bar{\alpha} X_{r} \cdots X_{2} X_{1}$ is symmetric ( $\alpha$ complex and the $X_{i}$ in $\mathcal{L}$ ), and $T$ is the sum of such operators. It follows that $u^{\prime}=0$, so that $P$ vanishes on the dense set $\mathscr{D}$ and hence is zero, that is, $\mathcal{X}=0$.

This theorem allows one of the hypotheses of a theorem in [2] to be eliminated, and in fact the following slightly stronger result can now be stated. The notation and hypotheses are those of the preceding theorem.

COROLLARY. If the weakly closed algebra of operators generated by the $U(a), a \in G$, is a factor, then $d U(p)$ is the operation of multiplication by a scalar.

Set $T=d U(p)$ and let $V$ be an arbitrary unitary operator in the commutor $A^{\prime}$ of the weakly closed (necessarily self-adjoint) algebra of operators generated by the $U(a)$. Using again the fact that continuous linear operations commute with strong integration, it follows that $\mathcal{D}$ is invariant under $V$, and in fact if $u \in \mathcal{D}$ has the form $u=\int U(a) x f(a) d a$, then $V u=\int U(a) V x f(a) d a$. Observe next that $T$ commutes with $V$. To see this it is sufficient to note that every monomial in the $d U(X)$ with $X \in \mathcal{L}$ commutes with $V$. Now letting $X_{1}, \cdots, X_{r}$ be arbitrary in $\mathcal{L}$, then as shown in [2] $d U\left(X_{1} \cdots X_{r}\right) u$ $=\int U(a) x\left(X_{1} \cdots X_{r} f\right)(a) d a$, and it follows that $\operatorname{VdU}\left(X_{1} \cdots X_{r}\right) u$ $=\int U(a) V x\left(X_{1} \cdots X_{r} f\right)(a)=d U\left(X_{1} \cdots X_{r}\right) V u$. Hence the closure $T^{*}$ of $T$ likewise commutes with $V$, and so also do the projections 
$E_{\lambda}$ in the spectral resolution of $T^{*}$. It results that the $E_{\lambda}$ are all in $\mathcal{A}^{\prime \prime}=\mathcal{A}$.

To conclude the proof, it suffices to show that the $E_{\lambda}$ are also in $\mathcal{A}^{\prime}$, for as $A \cap \mathcal{A}^{\prime}$ consists only of scalar multiples of $I$, it then follows that $E_{\mu+0}=I$ and $E_{\mu-0}=0$ for some real $\mu$, so that $T^{*}=\mu I$. Now as shown in the proof of the theorem, $T$ commutes with the $U(a)$. Hence $T^{*}$ likewise commutes with the $U(a)$, and so also do the $E_{\lambda}$. Since the $U(a)$ generate $\mathcal{A}$, it results that $E_{\lambda} \in \mathcal{A}^{\prime}$.

\section{REFERENCES}

1. G. Birkhoff, Representability of Lie algebras and Lie groups by matrices, Ann. of Math. vol. 38 (1937) pp. 526-533.

2. I. E. Segal, $A$ class of operator algebras which are determined by groups, Duke Math. J. vol. 18 (1951) pp. 221-265.

3. I. M. Singer, Lie algebras of unbounded operators, to appear soon.

4. J. von Neumann, Allgemeine Eigenwerttheorie Hermitescher Funktional-operatoren, Math. Ann. vol. 102 (1929) pp. 49-131.

University of Chicago 